A N N A L E S Annales de Bretagne et des Pays de l'Ouest

Anjou. Maine. Poitou-Charente. Touraine

111-4 | 2004

Varia

\title{
Les insurrections girondistes de Bretagne en 1793 : premiers résultats
}

\section{Anne De Mathan}

\section{(2) OpenEdition}

\section{Journals}

Édition électronique

URL : http://journals.openedition.org/abpo/1171

DOI : $10.4000 / a b p o .1171$

ISBN : 978-2-7535-1496-6

ISSN : 2108-6443

Éditeur

Presses universitaires de Rennes

Édition imprimée

Date de publication : 20 décembre 2004

Pagination : 29-42

ISBN : 978-2-7535-0082-2

ISSN : 0399-0826

\section{Référence électronique}

Anne De Mathan, «Les insurrections girondistes de Bretagne en 1793 : premiers résultats », Annales de Bretagne et des Pays de l'Ouest [En ligne], 111-4 | 2004, mis en ligne le 20 décembre 2006, consulté le 02 mai 2019. URL : http://journals.openedition.org/abpo/1171 ; DOI : 10.4000/abpo.1171 


\title{
Les insurrections girondistes de Bretagne en 1793 : premiers résultats
}

\author{
Anne de Mathan \\ Maître de conférences, université de Bretagne Occidentale (Brest)
}

Pas toujours encline à envisager le sort des vaincus, l'histoire s'est longtemps montrée ingrate envers les Girondins et leurs partisans. Les bois de la guillotine démontés, les assignats démonétisés, l'Empire démantelé et les Bourbons restaurés, personne, en Bretagne comme en Gironde ${ }^{1}$, ne semblait disposé, en effet, à évoquer ces républicains modérés qui voulurent combattre l'hégémonie montagnarde. Ce n'est qu'après la Révolution de 1830 que les travaux d'Armand du Châtellier, Prosper Levot et René Kerviler ont commencé d'exhumer le mouvement de $1793^{2}$. Des érudits ont poursuivi l'effort de clarification et de publication de documents ${ }^{3}$. Le bicentenaire a suscité des monographies de notabilités finistériennes au cœur de l'affaire, comme l'évêque constitutionnel Alexandre Expilly ou le médecin Jean-Jacques Bouëstard de la Touche ${ }^{4}$. Mais la recherche universitaire n'a envisagé cette question que dans des mémoires de maîtrise ${ }^{5}$ ou dans de plus vastes synthèses ${ }^{6}$. En Ille-et-Vilaine, les histoires régionales ne font

1. Mathan, Anne de, Les " Hommes de la Gironde ». Acteurs, enjeux et modalités de l'insurrection de 1793, thèse, Université de Bordeaux III, 2000, et Lille, Presses Universitaires du Septentrion, 2 volumes, 2001.

2. Chatellier, Armand du, Histoire de la Révolution en Bretagne (1836), rééd. Morvran, 5 vol., 1978 et Brest et le Finistère sous la Terreur, Brest, 1858; LeVoT, Prosper, Histoire de la ville et du port de Brest pendant la Révolution, Brest, Lefournier, 1864-1866; KERVILER, René, La Bretagne pendant la Révolution Française, Société des Bibliophiles Bretons, Rennes, 1912.

3. BERnARD, Daniel, "Le procès des administrateurs du Finistère devant le tribunal révolutionnaire de Brest ", Cahiers de l'Iroise, 1961, p. 210-212, et 1963, p. 81-86.

4. L'HERRON, Jeanine, Un morlaisien dans la Révolution française, Louis Alexandre Expilly, recteur de Saint-Martin, Morlaix, Dossen, 1989; Stofft, Henri et SEgalen, Jean, Bouëstard, médecin-philosphe, franc-maçon jacobin, Morlaix, Dossen, 1989.

5. LE TENDRE, Philippe, L'insurrection fédéraliste du département du Finistère, Rennes 2, 1975, RocuET, Brigitte, Le fédéralisme à Brest pendant la période révolutionnaire (17921793), Université de Bretagne Occidentale, 1978, et DeLIN, Alexandre, L'insurrection girondiste du département du Finistère (1793-1794), Université de Bretagne Occidentale, 2001.

6. Le Gallo, Yves (dir.), Histoire de Brest, Toulouse, Privat, 1976, Le Finistère de la préhistoire à nos jours, Saint-Jean-d'Angély, Bordessoules, 1991; HEnwood, Philippe et 
qu'effleurer le sujet ${ }^{7}$, et depuis les articles de Daniel Stone en $1971^{8}$ et de Roger Dupuy en $1975^{9}$, aucune recherche n'a été menée sur ce sujet. À ce jour donc, les insurrections de quatre-vingt-treize en Bretagne n'ont fait l'objet d'aucune synthèse. Une quinzaine d'années après le colloque de Saint-Brieuc ${ }^{10}$, les Bleus semblent toujours éclipsés par les Blancs, et les Girondistes s'effacent derrière les Chouans. Il suffit pour s'en convaincre de jeter un œil sur les cartes proposées par les manuels universitaires pour s'apercevoir qu'en Bretagne, il n'y aurait que des rebelles et des brigands, quand ailleurs les insurrections départementales sont encore qualifiées de "fédéralistes ${ }^{11}$ ".

La Gironde a certes déploré la perte d'orateurs brillants comme Pierre Vergniaud ou Élie Guadet. Mais le Finistère refusa aussi la proscription de Jean-René Gomaire et Augustin Le Goazre de Kervélégan, tandis que l'Illeet-Vilaine combattait celle de Jean-Denis Lanjuinais. Ainsi la Bretagne eut ses Girondins, et ses Girondistes dans la personne des provinciaux qui les défendirent. La question présente un intérêt historiographique évident : la recherche sur les assises locales du parti girondin permet de connaître l'électorat que les députés représentent à l'Assemblée, afin de mesurer, d'une part, la coïncidence entre la politique des Girondins et les attentes de leurs commettants, et, d'autre part, le poids de l'antagonisme entre les intérêts provinciaux et les revendications des sans-culottes, dans les dissensions de la Convention. Elle permet aussi de comprendre les raisons de l'échec d'une révolte qui a secoué une soixantaine de départements et aurait pu constituer une grave menace pour la Convention jacobine.

Il s'agit ici d'un bilan d'étape, dans le cadre d'une recherche plus vaste et encore inaboutie, portant sur l'ensemble des départements de l'Ouest de la France. Pour l'heure, l'état des dépouillements, quasiment terminés dans le Finistère et l'Ille-et-Vilaine, permet de montrer les spécificités des acteurs, des enjeux et des modalités d'une insurrection d'importance nationale.

MonAnge, Edmond, Brest, un port en Révolution : 1789-1799, Rennes, Ouest-France, 1989 ; Kerherve, Jean, Histoire de Quimper, Toulouse, Privat, 1994; CloITRE, Marie-Thérèse (dir.), Histoire de Brest, Brest, CRBC, 2000.

7. Delumeau, Jean, Histoire de la Bretagne, Toulouse, Privat, 1969; MeYer, Jean (dir.), Histoire de Rennes, Toulouse, Privat, 1972; LEBRUn, François (dir.), L'Ille-et-Vilaine, des origines à nos jours, Saint-Jean-d'Angély, Bordessoules, 1984.

8. Stone, D., "La révolte fédéraliste à Rennes ", Annales Historiques de la Révolution Française, juillet-septembre 1971, $\mathrm{n}^{\circ} 205$.

9. Dupuy, Roger, "Aux origines du "fédéralisme" breton : le cas de Rennes, 1789-mai 1793 ", Annales de Bretagne et des pays de l’Ouest, 1975, nº 82, p. 337 sqq.

10. Actes du colloque Les Bleus de Bretagne, de la Révolution à nos jours, Fédération Côtes-du-Nord 1989, Saint-Brieuc, 1991.

11. Jessenne, Jean-Pierre, Révolution et Empire, 1783-1815, Paris, Hachette, collection Carré Histoire, 1993, p. 143. 


\section{Acteurs}

En Ille-et-Vilaine, la réunion des corps administratifs de Rennes, en avril 1793, face au péril blanc, sert de cadre à la rébellion girondiste ${ }^{12}$. Dans le Finistère, le département convoque à ses côtés les autorités secondaires après l'arrestation des députés girondins ${ }^{13}$. L'exercice d'un mandat public à cette époque, une mission à Paris, Laval, Rennes, Caen, ou l'engagement dans la force départementale, attestent la participation au mouvement girondiste. L'arrestation pour fédéralisme, les justifications politiques des prisonniers et, à Brest seulement, le jugement par le tribunal révolutionnaire, complètent le corpus des girondistes ${ }^{14}$. Au total, la documentation révèle les traces de 141 girondistes à Brest et 138 à Rennes.

La prosopographie des girondistes du Finistère et d'Ille-et-Vilaine, montre une certaine uniformité par-delà les diversités régionales ou locales. Les insurgés du Finistère ont un âge moyen d'environ 43 ans, et ceux d'Illeet-Vilaine d'environ 45 ans, soit un résultat très comparable à la moyenne d'âge de 42 années et demie observée en Gironde, et à Marseille où l'âge moyen oscille entre 43 et 44 ans. Ces résultats n'ont rien de révolutionnaire dans la mesure où ils confirment ce que l'on sait de longue date, depuis les travaux de Mathiez ${ }^{15}$ et Soboul ${ }^{16}$, Raymonde Monnier ${ }^{17}$, Michel Vovelle ${ }^{18}$, Françoise Brunel, Serge Bianchi ${ }^{19}$, Marcel Dorigny ${ }^{20}$, Maurice Genty et Émile Ducoudray $^{21}$ : l'engagement politique, quelle que soit sa coloration, est le fait de citoyens qui, dans la force de l'âge, installés à la fois dans leur vie professionnelle et familiale, ont à cœur de protéger acquis et valeurs politiques. Plus des deux tiers des insurgés du Finistère sont originaires du

12. Arch. dép. d'Ille-et-Vilaine, L 217.

13. Arch. dép. du Finistère, 3 L 3, Registres des délibérations du conseil général du département (décembre 1792-juillet 1793), 10 L 15, Bulletin du département du Finistère.

14. Arch. dép. du Finistère, Sous-série 8 L, Actes des représentants en mission, 10 L 125134, Police générale, détenus et suspects, 10 L 135, Poursuites contre les administrateurs du Finistère, 66 L 1, Jugements du Tribunal révolutionnaire. Arch. dép. d'Ille-et-Vilaine, L 1474, Comités de surveillance, Liste des suspects détenus à Rennes, 1793 - an III. L 1475, id., Pétitions adressées par les suspects détenus dans les prisons de Rennes, 1793 - an III, L 1480, id., Suspects détenus : pétitions en vue de leur libération, 1793 - an III, L 1480, id., Suspects détenus : listes, motifs d'arrestation, perquisitions, interrogatoires, 1793-an III. L 1482-92, id., Suspects détenus : dossiers individuels par ordre alphabétique.

15. Mathiez, Albert, Girondins et Montagnards, Paris, Armand Colin, 1930.

16. Soвoul, Albert, Les sans-culottes parisiens : mouvement populaire et gouvernement révolutionnaire (1793-1794), Paris, Clavreuil, 1958, rééd. Le Seuil, 1973, Les sans-culottes, Paris, Seuil, 1968, et (dir.), Girondins et Montagnards, Paris, Société des Études Robespierristes, 1980.

17. Soboul, Albert et MonNiER, Raymonde, Répertoire du personnel sectionnaire parisien en l'an II, Paris, Publications de la Sorbonne, 1985.

18. VovELL, Michel, « Le sans-culotte marseillais », Histoire et Mesure, n 1, 1986, p. 93-103.

19. BianchI, Serge, La Révolution culturelle de l'an II : élites et peuple (1789-1799), Paris, Aubier, 1982.

20. DoRIGny, Marcel, Les Girondins et le libéralisme économique, thèse, Paris I, 1992.

21. GENTY, Maurice, "Les élections parisiennes de 1789 à 1792. Étude socioprofessionnelle des élus locaux ", Ibid., p. 59-70; DucoudRAY, Émile, " Bourgeois parisiens en révolution, 1790-1792", ibid., p. 71-88. 
département. Le tiers des insurgés d'Ille-et-Vilaine est né à Rennes. Ces mesures sont semblables aux résultats obtenus en Gironde. Ceci corrobore une sorte d'invariant de la société du temps, marquée par l'assez faible mobilité géographique des hommes. Cela indique aussi un fort enracinement local qui induit un sentiment de fraternité à l'égard des compatriotes proscrits à Paris, mais n'empêche pas l'implication politique dans une question nationale.

Les professions apportent un peu de variété au sein de ce milieu provincial. Les Girondistes finistériens ressemblent, à cet égard, à ceux de Gironde.

\section{Les professions des insurgés de Gironde}

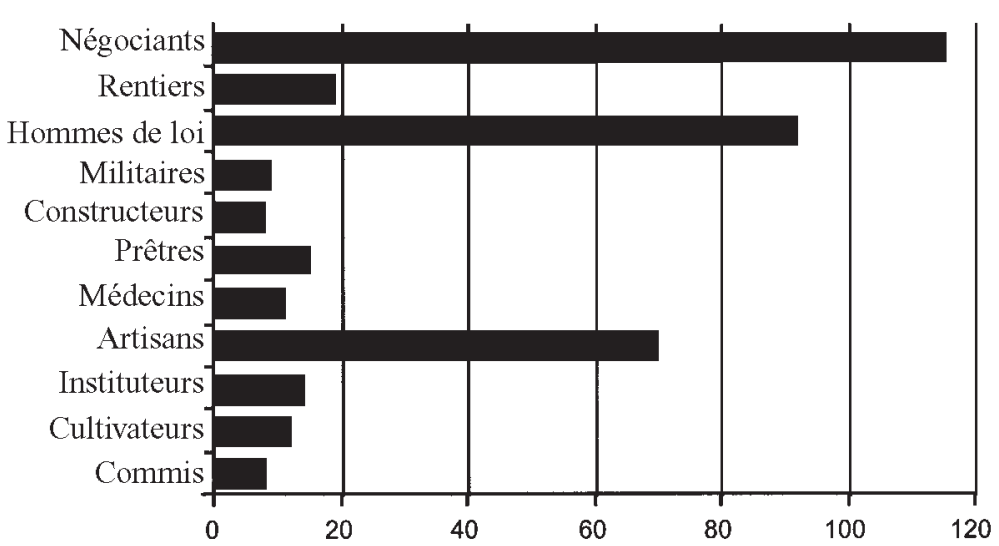

Les négociants y sont certes moins nombreux qu'à Bordeaux ou qu'à Marseille, où le grand commerce se pratique sur une autre échelle. Mais la bourgeoisie des affaires regroupant le comptoir et la boutique, l'échoppe et la manufacture, y représente néanmoins le tiers des effectifs. Et le monde de la basoche, formé d'avocats et de quelques notaires, concerne à lui seul un tiers des professions. Les militaires, surtout officiers de marine ou cadres des douanes, représentent un cinquième des effectifs. Parmi les quatre ecclésiastiques, trois prêtres sont jureurs, dont l'évêque constitutionnel Alexandre Expilly, et un curé s'est défroqué.

À Rennes, en revanche, le monde du commerce est quasiment absent, avec seulement trois négociants ou fournisseurs aux armées et trois artisans et commerçants. La judicature exerce une incontestable domination sur cette insurrection dont les deux tiers des acteurs sont avocats, notaires et procureurs. Cette sur représentation s'explique évidemment par les activités de la ville où siégeait le Parlement de Bretagne. Pourtant Bordeaux avait le Parlement de Guyenne, et seul un cinquième d'hommes de loi formaient les rangs de l'insurrection. Ces variations ne s'expliquent guère que par la composition sociale des villes et ses effets sur les spécificités locales 


\section{Les professions des girondistes du Finistère}

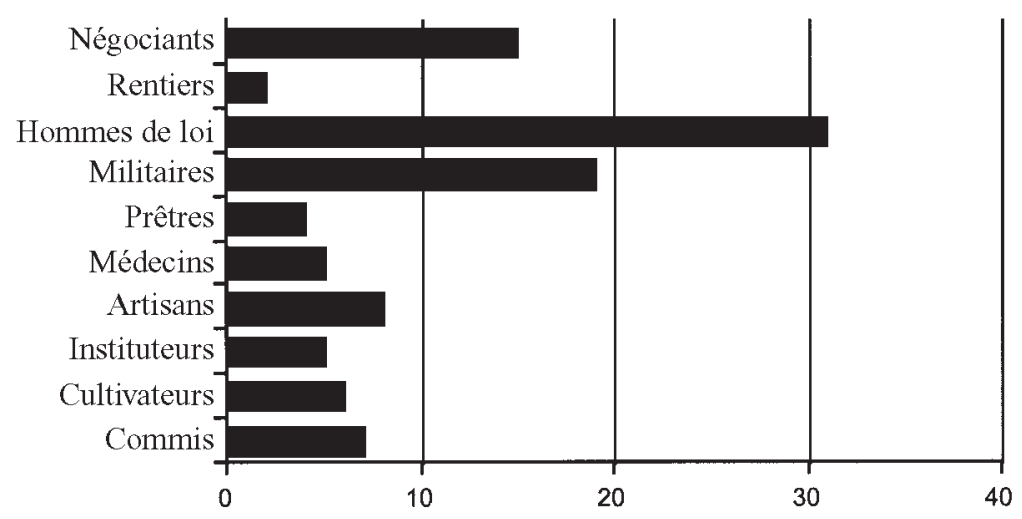

de l'événement révolutionnaire. A Bordeaux, aux débuts de la Révolution, la découverte du politique et l'accès aux magistratures locales fut, dans une relative harmonie avec les élites de l'Ancien Régime, le fait du négoce, tandis qu'à Rennes, ce fut davantage la "robinocratie ", plus tard conspuée par Carrier, qui dirigea la scission entre les ordres et l'autonomisation politique du haut tiers face aux nobles bretons ${ }^{22}$.

\section{Les professions des girondistes d'Ille-et-Vilaine}

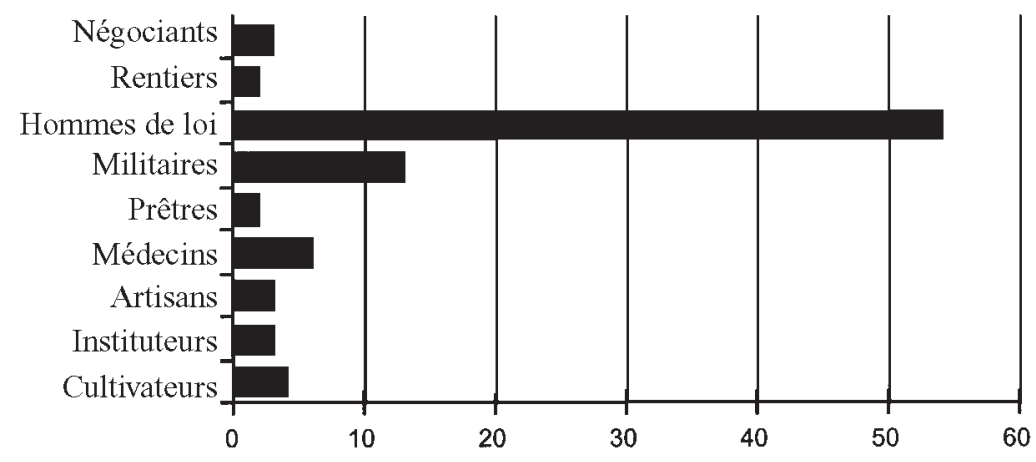

En 1793, les militaires sont assez présents à Rennes où ils participent aux séances des corps administratifs réunis. En effet, les troubles de la

22. LHERITIER, Michel, La Révolution à Bordeaux, tome 1, La fin de l'Ancien Régime, tome 2, Liberté, Paris, Daubin, 1942-1947; Coste, Laurent, La difficile gestion municipale d'une grande ville sous l'Empire : Bordeaux de 1805 à 1815, thèse, Bordeaux III, 1990; FIGEAC, Michel (dir.), Histoire des Bordelais, tome 1, La modernité triomphante (1715-1815), Bordeaux, Mollat-Fédération Historique du Sud-Ouest, 2002; DENIS, Michel, Rennes, berceau de la Liberté. Révolution et démocratie. Une ville à l'avant-garde, Rennes, OuestFrance, 1989. 
chouannerie agitent alors les campagnes, et particulièrement les districts du sud du département. Mobilisés dans le combat contre les Blancs, ces militaires républicains n'approuvent pas pour autant l'élimination des Girondins qu'ils ne considèrent manifestement pas comme un obstacle à la politique du salut public. Parmi les deux ecclésiastiques rennais, se trouvent enfin le curé jureur Joseph Lanjuinais, frère du député, et l'évêque constitutionnel Claude Le Coz.

Les acteurs des insurrections bretonnes se caractérisent enfin par le fait que, dans une écrasante majorité, ils exercent des responsabilités publiques dans les administrations locales. Dans le Finistère, plus des deux tiers des insurgés sont, en effet, des fonctionnaires. Quelques-uns ont participé à la vie politique nationale comme le négociant François Le Déan, député de Quimper aux États généraux ou Blaise Cavellier, commis de la marine à Brest et député à la Législative. La faiblesse de l'implication dans les affaires publiques après Thermidor montre que l'expérience traumatisante de l'an II a détourné beaucoup de patriotes de l'engagement politique. Néanmoins, une dizaine de rescapés de la Terreur poursuivent leur carrière. Louis Roujoux, avocat au Parlement de Rennes sous l'Ancien Régime, député à la Législative, est accusateur public du tribunal criminel du département, et aux premiers rangs de l'insurrection girondiste. Réintégré dans ses fonctions en 1795, il entre au Conseil des Anciens où il est continué en 1798. Préfet de Saône-et-Loire en 1802, décoré de la légion d'honneur en 1804, il est fait baron d'Empire l'année suivante. Préfet d'Eure-et-Loire pendant les Cents Jours, il rentre dans la vie privée à la Restauration et meurt en 1829 .

Plus encore que dans le Finistère, l'insurrection rennaise est le fait de ses magistrats publics qui constituent plus des quatre cinquièmes des effectifs. Quelques exemples illustrent la possibilité d'une continuité entre les carrières publiques de l'Ancien Régime et celles de la France révolutionnaire, à l'instar de Joseph Talhouet. Président à mortier du Parlement de Bretagne, ce ci-devant noble devient maire de Rennes au début de la Révolution. Administrateur du département, il participe activement à la révolte de 1793. Au moins sept rennais poursuivent aussi une carrière nationale, comme Joseph Malherbe, procureur général syndic du département en 1793 ou encore Bouaissier, président du tribunal criminel du département, qui entrent au conseil des Cinq Cents en l'an VIII.

À Rennes, la Terreur a été moins sévère qu'ailleurs à l'encontre des coupables de fédéralisme. Contrairement à Brest et à Bordeaux, un assez faible nombre d'arrestation semble y avoir été ordonné pour simple opinion favorable au fédéralisme. En revanche, plus de la moitié des girondistes du Finistère ont été arrêtés et incarcérés, et trente d'entre eux, dont les vingtsix administrateurs du département, ont péri sous la guillotine, soit le quart des effectifs de l'insurrection. En Gironde, soixante-huit personnes payèrent de leur vie leur fidélité envers les députés Girondins, mais ces victimes constituaient moins d'un sixième du corpus. La relative modération de la Terreur bordelaise s'explique notamment par la personnalité des repré- 
sentants du peuple Ysabeau et Tallien. Mais à Brest, Jean Bon Saint-André eut à compter avec les jacobins radicaux qui formèrent un tribunal révolutionnaire contre son gré, et pour qui l'enjeu stratégique du port, après la trahison de Toulon, la proximité de la Vendée et les tentations contre-révolutionnaires des campagnes léonardes constituaient autant d'arguments à la Terreur. En Ille-et-Vilaine en revanche, moins de la moitié des girondistes ont été arrêtés et seulement les deux tiers étaient des élus alors que ces derniers constituaient plus des deux cinquièmes du corpus. Aucun d'entre eux ne fut jugé par les commissions militaires qui se sont uniquement occupées des contre-révolutionnaires.

Représentants dans la force de l'âge d'une moyenne bourgeoisie des talents dominée par la judicature, très impliqués dans la vie politique locale, les Girondistes du Finistère qui payèrent un lourd tribut à la Terreur, et ceux d'Ille-et-Vilaine qui furent davantage épargnés, ressemblent à bien des égards aux acteurs de l'insurrection bordelaise.

\section{Enjeux}

Les enjeux idéologiques qui motivent les révoltes anti-jacobines révèlent aussi une certaine stabilité d'une région à l'autre. On retrouve en Bretagne cette conception à géométrie variable de la légalité républicaine qui s'outrage du coup de force du 2 juin 1793, mais légitime le choix de l'insurrection par le droit de résistance à l'oppression. Dès les premières dissensions de la Convention à l'automne 1792, les administrateurs du Finistère analysent la situation par une alternative marquée : aux Montagnards, "ces hommes de sang ${ }^{23}$ ", " ces monstres sanguinaires et perfides ${ }^{24}$ ", secondés par les sans-culottes, "ce ramas de factieux stipendiés par un parti secret et, peut-être, par des despotes étrangers ${ }^{25}$ ", s'opposent, dans une parfaite symétrie, les députés girondins, " hommes probes ${ }^{26}$ " dont les " vertus " et les « lumières " méritent la « confiance ${ }^{27}$ " des girondistes finistériens, " vrais amis de la liberté ${ }^{28}$ ». Avant même le 2 juin, les corps administratifs de Rennes déplorent l'antagonisme des partis et déclarent à la Convention, le 11 mai, " nous ne connaissons ni ne voulons connaître Robespierre ni Guadet, Danton ni Gensonné, ni la Montagne, ni la Vallée, ni toutes ces lignes de démarcation qui dégradent la dignité de représentants d'une nation

23. Adresse du département aux 48 sections de Paris, le 16 octobre 1792 (Arch. dép. du Finistère, 10 L 135).

24. Arrêté du département, le 15 décembre 1792, cité par Armand du Châtellier, op. cit., p. $70-71$.

25. Adresse du département à la Convention, le 29 décembre 1792 (Arch. dép. de la Gironde, 10 L 135).

26. Adresse du Finistère aux autres départements de Bretagne, le 14 juin (Armand du Châtellier, op. cit., p. 79-82).

27. Adresse aux sections et aux départements de Paris, le 12 juin (Armand du Châtellier, op. cit., p. 78).

28. Bulletin du département du Finistère, 8 juin 1793 (Arch. dép. du Finistère, 10 L 15). 
libre ${ }^{29}$ ". Pour autant, les Rennais condamnent sans ambiguïté le projet d'éliminer les Girondins : "Les anarchistes ", préviennent-ils, " ne peuvent se déguiser au regard des vrais républicains. Les anarchistes sont à nos yeux ceux qui sollicitent la dissolution de la Convention nationale ${ }^{30}$ ".

Les événements des 31 mai-2 juin sont dénoncés en Bretagne avec les mots de la passion politique et de l'émotion morale. La première réaction des administrateurs du Finistère qui écrivent à l'Assemblée nationale témoigne ce registre affectif. "Au milieu des baïonnettes, au milieu du fer des assassins, au milieu des cris de rage des soudoyés de Pitt et Cobourg ", reprochent-ils aux législateurs, " vous avez manqué d'attacher à vos noms une gloire immortelle. Insensibles à l'honneur de mourir à votre poste, vous avez avili votre caractère et dégradé la représentation nationale ${ }^{31}$ ". De même, les citoyens des sections de Rennes se livrent à une extraordinaire analyse des faits, toute de bruit et de fureur :

"Le 10 mars, cette faction tente de faire égorger au sein même de la Convention nationale ceux des Représentants dont elle avait à craindre les lumières et l'intégrité. L'infâme projet échoue. [...] Une Commission est créée pour suivre et dévoiler cette conspiration ourdie contre la liberté publique et la représentation nationale. [...] Les factieux mettent sous le joug une partie des Sections de Paris, s'emparent de l'autre, l'entraînent à la Convention, en arrachant un décret qui dissout cette Commission. Le lendemain, le décret est rapporté; ils n'en deviennent que plus audacieux; le tocsin sonne, le canon d'alarme se fait entendre de toutes parts; et si ce jour même ils ne consomment pas leur horrible attentat, c'est que tout Paris est levé... C'est que la masse du peuple est pure... Ils poursuivent; deux jours après, les victimes désignées sont en leur puissance. Ici s'accumulent des violations inouïes jusqu'alors de tous les principes. Ici se développe toute l'horreur du plus noir complot. Les droits les plus sacrés de l'homme et du citoyen sont méconnus; la majesté nationale est outragée; la liberté, la loi publique sont frappées jusque dans leur dernier asile [...]. Toute communication est rompue entre Paris et les Départements. On isole Paris de la République entière, et dans cet état de révolte d'une faction scélérate contre l'unité, l'indivisibilité de la République, dans ce bouleversement épouvantable de toutes les lois, la France doute si ses Représentants vivent encore ${ }^{32}$."

Dans cette situation, le conseil général d'Ille-et-Vilaine déclare le 6 juin :

" qu'au milieu des orages qui s'élèvent contre la République Française, il ne voit [...] que le moyen de la sauver par un heureux concours de tous les Départements contre les cruels et les perfides anarchistes qui la déchirent et [...] que la liberté ne renaît et ne se conserve que par la plus active résistance à l'oppression ${ }^{33}$ ".

29. Arch. dép. d'llle-et-Vilaine, L 217.

30. Arch. dép. d'Ille-et-Vilaine, L 217.

31. Armand du Châtellier, op. cit., p. 78.

32. Arch. dép. d'Ille-et-Vilaine, L 223.

33. Arch. dép. d'Ille-et-Vilaine, L 223. 
Les jours passant, le discours se théorise davantage. Louis Roujoux, président de l'assemblée centrale de résistance à l'oppression de Caen, explique que :

"Les droits de l'homme ont été méconnus, notamment dans l'article 2 qui porte : "Le but de toute association politique est la conservation des droits naturels et imprescriptibles de l'homme. Ces droits sont la liberté, l'égalité, la sûreté des personnes et la résistance à l'oppression" et dans l'article 6 qui porte : "La loi est l'expression de la volonté générale. Tous les citoyens ont le droit de concourir, personnellement ou par leurs représentants, à sa formation. Elle doit être la même pour tous, soit qu'elle protège, soit qu'elle punisse, etc. ${ }^{34}$." "

L'argument constitutionnel est pertinent. L'arrestation des Girondins sans rapport d'accusation est contraire aux droits de l'homme et à la souveraineté du peuple qui n'est plus entièrement représenté à l'Assemblée. La violation des principes démocratiques rompt le pacte social unissant les Français. Le droit de résistance à l'oppression leur fait un devoir de s'insurger contre ce coup de force. Mais les Girondistes se réfèrent à la Déclaration des droits de 1789 et à la Constitution de 1791, sans valeur légale sous la République. Les Montagnards ont dès lors beau jeu d'opposer à cette légalité d'un autre temps, l'impératif du salut public qui légitime une politique d'exception dans le moment de crise que traverse la nation.

Les Girondistes étendent la condamnation du coup de force à toute la ville de Paris. Dès le 16 octobre, les administrateurs du Finistère dénoncent les sans-culottes qui ont "poussé l'audace jusqu'à menacer les départements, comme si la quatre-vingt-troisième portion de la République pouvait inspirer un sentiment de terreur à une nation entière ". Ils contestent à la Commune de Paris le rôle dirigeant qu'elle prétend exercer sur le cours de la Révolution, par cet avertissement très clair et cette allusion au rôle des fédérés finistériens dans la prise des Tuileries : "Songez qu'une seule ville ne fait pas la loi à toute la République; songez à qui appartient la gloire du 10-Août ${ }^{35}$. " Les administrateurs profèrent même cette menace au département et à la Commune de Paris : "Empêchez un crime de plus, ou craignez la prophétie d'Isnard ${ }^{36}$ ! " Le député des Bouches-du-Rhône avait en effet lancé à la barre de la Convention le 27 mai cette menace incendiaire :

"S'il arrivait [...] qu'on portât atteinte à la représentation nationale, je vous le déclare, $[\ldots]$ Paris serait anéanti. La France entière en tirerait une vengeance éclatante, et bientôt on chercherait sur les rives de la Seine si Paris a existé."

Cette violence verbale n'est pas habituelle aux insurgés du Finistère qui distinguent les sans-culottes du reste des Parisiens. Ils expliquent en effet que :

34. Arch. dép. du Finistère, 10 L 135, 2 juillet.

35. Arch. dép. du Finistère, 10 L 135.

36. Arch. dép. du Finistère, 3 L 3, 7 juin 1793. 
«Les phalanges guerrières que le patriotisme appelle des départements vers Paris, ne sont point armées pour combattre les citoyens de cette ville, mais au contraire pour s'unir à la saine portion de ses habitants, afin de cimenter ensemble l'unité et l'indivisibilité de la République, la sûreté des personnes et des propriétés, la liberté, l'égalité, la garantie sociale, le respect et l'inviolabilité des représentants du peuple ${ }^{37}$."

De même, à la question " quel est le devoir du peuple? ", les Rennais répondent clairement :

"Se lever tout entier, marcher à Paris, non pour le combattre [...], mais pour se rallier à des milliers de frères qui n'attendent que sa présence pour repousser l'oppression et rendre à la représentation nationale sa dignité, son intégrité, sa liberté ${ }^{38}$."

Plus encore qu'en Gironde, les Bretons veillent à ne pas donner de prise à l'accusation de fédéralisme. La devise du Bulletin du Finistère proclame "Unité et indivisibilité ". Le département explique à leurs concitoyens que le comité de correspondance formé à Laval ne prétend pas être représentatif, " à moins ", hypothèse impensable, " de renoncer au principe d'indivisibilité ${ }^{39}$ ". Les administrateurs poussent même la fiction jusqu'à se demander quel serait alors le rôle de l'armée et craignent que la France déchirée tombe à la merci des généraux. Ils concluent que l'insurrection doit se borner à tout tenter pour réintégrer la Convention. Ils envoient à Rennes des commissaires à qui ils font la recommandation suivante :

"Réunis pour sauver la République, vous devez porter le comité central de correspondance hors le territoire de la ci-devant province de Bretagne, afin d'en écarter tout soupçon de vouloir s'isoler du reste de la République ${ }^{40}$. "

Les corps administratifs de Rennes veulent aussi se prémunir de cette accusation, et dès le 10 juin,

" un membre observe que le vœu de l'insurrection, quoique unanimement et spontanément exprimé, pourrait être présenté par des malveillants comme un système de fédéralisme concerté entre des départements, districts et communes de la ci-devant province de Bretagne et autres départements voisins, que pour prévenir toute injuste inculpation à cet égard, il propose de renouveler à l'instant les serments à l'égalité, à la liberté et à la République une et indivisible."

Ce qui est aussitôt fait ${ }^{41}$.

Républicains et patriotes, attachés à la défense des droits de l'homme et à la souveraineté de la nation, hostiles aux sans-culottes parisiens mais nullement fédéralistes, les Girondistes du Finistère et d'Ille-et-Vilaine légitiment leur insurrection par le droit de résistance à l'oppression et enten-

37. Arch. dép. du Finistère, 3 L 3, 21 juin 1793.

38. Arch. dép. d'Ille-et-Vilaine, L223.

39. Armand du Châtellier, op. cit., p. 79-82.

40. Ibid., p. 86-87.

41. Arch. dép. du Gers, L 223, 10 juin 1793. 
dent travailler dans une sorte d'eschatologie constitutionnelle au " bonheur de la génération actuelle et des générations futures ${ }^{42}$ ". En revanche, les revendications si puissantes en Gironde autour de la sûreté des propriétés et de la liberté économique ne trouvent aucun écho en Bretagne, ce qui s'explique en partie par la composition sociale du mouvement.

\section{Modalités}

La participation du bataillon du Finistère à la prise des Tuileries contribue sans doute à forger en Bretagne l'illusion de la toute-puissance des interventions départementales. Dès le 11 décembre 1792, l'administration du Finistère envoie six cents hommes pour protéger la Convention. Ils participent au maintien de l'ordre lors des pillages en février, puis rentrent au pays sans bruit. Alarmé des divisions persistantes de l'Assemblée, le département réitère cette mesure, le 30 mai 1793 :

"Considérant [...] qu'il est plus que temps enfin de balayer la terre de la liberté de cette minorité impure qui, par la réunion de tous les crimes, prétend dicter des lois au souverain, asservir ses représentants les plus fidèles et dissoudre à la fois la Convention et la République, [il] arrête la levée d'une force départementale de quatre cents hommes, à l'effet de se rendre incessamment à Paris, pour y protéger la sûreté de la Convention et celle des personnes, des propriétés et de la fortune publique ${ }^{43}$."

En Ille-et-Vilaine, la décision d'envoyer une force départementale est prise le 31 mai, avant même l'arrestation des Girondins. Dès le lendemain de cette nouvelle arrivée à Rennes le 5 juin, les corps administratifs réunis arrêtent que :

"Les vrais et courageux Patriotes, amis incorruptibles de la République et tous ceux qui, détestant l'horrible anarchie, ont dans l'âme la volonté ferme, constante et inébranlable d'en arrêter les progrès meurtriers sont inviter à composer, d'un moment à l'autre et de concert avec les autres Départements de la France, une Force armée dont la destination est de se porter dans le plus bref délai à Paris, pour retirer la Convention nationale de l'état d'oppression où la tiennent les anarchistes, assurer la Liberté individuelle des membres qui la composent, ou la leur recouvrer, obtenir la plus prompte et la plus sévère punition des désorganisateurs qui en arrêtent la marche, et protéger la sûreté des personnes, des propriétés et de la fortune publique $^{44}$."

La bannière de l'armée du Finistère est celle qu'arboraient les premiers fédérés dans « la touchante alliance entre Paris et les départements lors de la fête immortelle du 14 juillet $1790^{45}$ ", et dont les signes de la royauté ont été effacés. Le drapeau du bataillon d'Ille-et-Vilaine est " tricolore, sous l'enseigne de la République Française, avec la double inscription Liberté, Éga-

42. A. du Châtellier, op. cit., p. 125.

43. Arch. dép. du Finistère, 3 L 3.

44. Arch. dép. d'Ille-et-Vilaine, L 223, 6 juin 1793.

45. Arch. dép. du Finistère, 3 L 3, 21 juin 1793. 
lité, d'un côté; et de l'autre, Haine à la royauté et à l'anarchie ${ }^{46}$ ". Les Fédérés du Finistère partent le 23 juin sous le commandement de Jean-Noël Souché de la Brémaudière, et arrivent à Rennes le 2 juillet. Ils rejoignent à Caen les forces des autres départements dont celles d'Ille-et-Vilaine et participent à la " bataille sans larmes " de Pacy-sur-Eure, le 13 juillet, où l'incurie du commandant des troupes girondistes le dispute au désordre des troupes de la Convention : le premier coup de canon provoque la débandade des deux camps.

Le 7 juin, le département du Finistère réuni aux autorités secondaires à Quimper avait arrêté l'envoi de dix commissaires à Paris " pour se rendre auprès de la Convention et y réclamer, avec toute l'énergie de la liberté et de la justice, l'entière inviolabilité des citoyens Kervélégan et Gomaire, et des autres membres de la Convention constitués en état d'arrestation ". Quand ces derniers arrivent à Paris, avec les commissaires que les autorités d'Ille-et-Vilaine leur ont adjoints, les opinions s'y révèlent très hostiles, et ils doivent s'enfuir en brûlant tous leurs papiers, afin d'échapper aux mandats d'arrêt. Le Finistère invite, le 14 juin, les Côtes-du-Nord, le Morbihan, l'Ille-et-Vilaine, et la Loire Inférieure à envoyer un député à Laval pour y former un comité de correspondance. Le comité s'installe en réalité à Rennes le 19 juin, où l'on prête, comme à Bordeaux, le serment de " faire une guerre éternelle aux tyrans, aux traîtres et aux anarchistes, de maintenir la liberté, l'égalité, la République une et indivisible, la sûreté des personnes et des propriétés, en même temps que la souveraineté nationale ". Les autorités constituées de Rennes invitent en outre les communes et les districts du département à envoyer des députés à ce comité central qui constitue une sorte de chambre consultative, quand les corps réunis de Rennes conservent la direction du mouvement. Puis le comité central s'exile hors de Bretagne et se fond dans l'assemblée centrale de résistance à l'oppression de Caen, composée de députés des départements, des districts et des communes du Calvados, de l'Eure, d'Ille-et-Vilaine, de la Loire Inférieure, du Morbihan, des Côtes-du-Nord et du Finistère. Outre la coordination des mouvements armés et la rédaction d'adresses incendiaires répandues dans toute la France, cette assemblée qui devient l'instance dirigeante des insurrections de l'Ouest, organise l'embargo sur l'approvisionnement de Paris.

Lorsque la Convention propose à la ratification populaire la Constitution tant réclamée par les Girondins et leurs partisans, l'assemblée de Caen prend encore une décision lourde de conséquence. "Sans doute ", ordonnet-elle aux Français,

" vous ne ferez pas aux courageux Bordelais, aux fiers Bretons, aux intrépides Normands, aux indomptables Marseillais, l'injure de croire qu'ils veulent autre chose que la liberté, l'anéantissement des factions, le règne de l'ordre et des lois. [...] Au nom de la patrie, au nom de la liberté qui nous a coûté tant de sacrifices, n'allez pas accepter une constitution [...] qu'ils ne

46. Arch. dép. d'Ille-et-Vilaine, L 223, 6 juin 1793. 
peuvent regarder comme l'ouvrage de leurs représentants, une constitution que dès lors ils ne pourraient accepter sans trahir leur serment de vivre libres ou de mourir, puisque cette acceptation serait un premier acte de soumission à la tyrannie ${ }^{47}$."

Les administrateurs du Finistère adoptent cette position et expliquent le 9 juillet aux municipalités des campagnes de son ressort que

"malgré l'intégrité de la représentation nationale violée, les partisans de l'anarchie n'ont pas rougi de brocher dans un court intervalle, un projet de constitution et de le présenter à la France entière ${ }^{48}$."

Ils décident de ne pas convoquer les assemblées primaires et de rédiger une instruction à leurs concitoyens sur la nécessité de différer l'acceptation de la constitution jusqu'à ce que les griefs des départements soient satisfaits. Ce faisant, ils aggravent considérablement leurs torts aux yeux de la Convention qui a beau jeu d'ajouter à l'accusation de tendre au fédéralisme celle de fomenter la contre-révolution. En Ille-et-Vilaine, les corps administratifs se montrent en revanche plus légalistes et décident le 12 juillet de convoquer les Assemblées primaires pour le référendum. Face aux premières rétractations qui, le 17 juillet, montrent que le doute s'insinue parmi les insurgés, les corps administratifs organisent en leur sein un appel nominal pour ou contre la continuation du mouvement.

Mais la nouvelle de la défaite de Pacy se répand, et le 19 juillet 1793, la Convention, sur rapport de Barère, décrète d'accusation dix-neuf administrateurs du Finistère " pour avoir tenté d'avilir la représentation nationale, d'usurper l'autorité du souverain et comme coupables d'entreprises contrerévolutionnaires ${ }^{49}$ ". Les huit administrateurs restés en fonction écrivent le 24 juillet à la Convention pour désavouer leur position sur la Constitution et passer sous les fourches caudines de la Montagne. Le 26 juillet, les corps administratifs de Rennes ordonnent le rappel de leur force départementale et envoient leur soumission à la Convention nationale.

Les insurgés bretons ont, comme dans les autres départements, péché par optimisme. Le temps est passé où les citoyens de Rennes tenaient ce langage audacieux à la Convention, " ce mouvement sera terrible; calculez-en les effets; hâtez-vous de les prévenir; rapportez l'odieux décret qui met en état d'arrestation nos plus incorruptibles défenseurs; rendez-les à la République; vous en répondez sur vos têtes ${ }^{50}$ ", et où les corps réunis assuraient que " le triomphe des bons Patriotes et des vrais Républicains ne peut qu'être assuré ${ }^{51}$ "! Grisés par le succès des fédérés dans la chute de la monarchie, ils n'ont pas saisi la puissance du mouvement populaire dont l'instrumentalisation par la Montagne a permis de réduire les oppositions à la Convention. Les Girondistes ont surestimé le degré de politisa-

47. Armand du Châtellier, p. 123-125.

48. Ibid., p. 125.

49. Ibid.

50. Arch. dép. d'Ille-et-Vilaine, L 223, 5 juin 1793.

51. Arch. dép. d'llle-et-Vilaine, L 223, 6 juin 1793. 
tion de leurs concitoyens, à une époque où l'abstention était pourtant massive. Ils ont surévalué la capacité de mobilisation après la levée des trois cent mille hommes, et au moment où ils parvenaient tout juste à contenir dans leurs propres départements les troubles contre-révolutionnaires.

La parenté des insurrections départementales apparaît donc frappante, dans leurs acteurs, leurs enjeux et leurs modalités. Organisée par une poignée d'honnêtes bourgeois, amis des droits de l'homme, de l'ordre et des lois, moins soucieux qu'en Gironde de leurs intérêts économiques, la rébellion bretonne ne parvient pas plus qu'ailleurs à s'attirer un vrai soutien populaire. La réaction des départements aux événements du 2 juin, apparaît bien loin des réalités politiques nationales, mais aussi locales. Et ce sont, sans doute, le manque de lucidité politique des insurgés de 1793, et l'inconséquence de leur révolte dirigée contre le pouvoir central, qui expliquent le désaveu infligé par leurs contemporains à leur entreprise et à leur postérité.

\section{RESUME}

Le 2 juin 1793, sous la pression des piques, des baïonnettes et des canons braqués sur elle, la Convention décrète au nom du salut public l'arrestation de trente-deux députés de sensibilité girondine. À la nouvelle de ce coup de force, les départements privés de représentation entrent en résistance. En Bretagne, les autorités constituées envisagent ensemble des mesures propres à restaurer à Paris l'ordre et la loi. Moins que les détails de l'insurrection consistant essentiellement dans la levée de forces départementales, il s'agit de repérer tenants et aboutissants d'une idéologie toute républicaine, mais hostile à la suspension de l'ordre constitutionnel malgré les circonstances, et d'analyser la composition sociologique d'un mouvement sans collusion avec la Contre-Révolution.

\section{ABSTRACT}

On $2^{\text {nd }}$ June 1793, faced with pikes, bayonets and guns aimed directly at them, the Convention ordered the arrest, in the name of public safety, of 32 deputies with Girondist sympathies. At the news of this takeover by force, the departments thus deprived of representation, began to offer resistance. In Brittany, an official body of authorities met to consider measures to restore law and order in Paris. Rather than analysing the details of the insurrection, which consisted mainly in the raising of departmental forces, the autorities sought to examine in depth an ideology, which although republican to the core, was hostile to the suspension of constitutional law, despite the circumstances and to analyse the sociological composition of a movement independent of the counter-revolution. 